\title{
Auditory and visual spatial working memory
}

\author{
GÜNTHER LEHNERT and HUBERT D. ZIMMER \\ Saarland University, Saarbrücken, Germany
}

\begin{abstract}
A series of experiments compared short-term memory for object locations in the auditory and visual modalities. The stimulus materials consisted of sounds and pictures presented at different locations in space. Items were presented in pure- or mixed-modality lists of increasing length. At test, participants responded to renewed presentation of the objects by indicating their original position. If two independent modality-specific and resource-limited short-term memories support the remembering of locations, memory performance should be higher in the mixed-modality than in the pure-modality condition. Yet, memory performance was the same for items in both types of list. In addition, responses to the memory load manipulation in both modalities showed very similar declines in performance. The results are interpreted in terms of object files binding object and location information in episodic working memory, independently of the input modality.
\end{abstract}

According to the tripartite working memory model (Baddeley \& Hitch, 1974; Baddeley \& Logie, 1999), humans have available two mechanisms for the temporary storage and maintenance of information: the phonological loop (PL) and the visual spatial sketchpad (VSSP). The VSSP is further subdivided into the visual cache and the inner scribe (see Logie, 1995). The cache is a passive store holding visual information, such as the color of objects, whereas the inner scribe is an active rehearsal process that refreshes information held in the cache. Furthermore, it is assumed that the visual cache stores not only visual but also static spatial information (e.g., the spatial layout of objects), whereas the inner scribe is involved in the maintenance of dynamic spatial information. Hence, in the tripartite model, the device for temporary storage of nondynamic spatial components is the visual cache (Logie, Engelkamp, Dehn, \& Rudkin, 2001). This is convincing as long as only visual spatial input is considered. However, spatial information is not only provided visually, but also by aural means. The source of a heard sound can usually be located quite easily. However, if the VSSP is purely visual working memory, as has been suggested,

This research was carried out within the Collaborative Research
Centre for Resource-Adaptive Cognitive Processes (SFB 378) through
a grant to H.D.Z. and Axel Mecklinger by the Deutsche Forschungs-
gemeinschaft. We thank Kristin Günther and Yvonne Pooch for their
help in running the experiments and Ullrich Ecker for native speaker
assistance. A portion of the manuscript was written while H.D.Z. was a
fellow of the Centre for Advanced Study at the Norwegian Academy of
Science and Letters, Oslo, Norway, within the research group "Towards
a Comprehensive Model of Human Memory." Correspondence concern-
ing this article should be addressed to G. Lehnert or H. D. Zimmer, De-
partment of Psychology, Saarland University, P.O. Box 151150, D-66041
Saarbrücken, Germany (g.lehnert@mx.uni-saarland.de or huzimmer@
mx.uni-saarland.de).

Note-This article was accepted by the previous editorial team, when Colin M. MacLeod was Editor. auditory input does not match its format. Thus, the challenging question arises of how spatial locations of sounds are temporarily remembered. The following experiments attempted to answer this question.

Auditory spatial information has been a topic in some previous working memory research. For example, in early experiments investigating the VSSP, auditory stimuli were also used, since at that point the mechanism was mainly considered a spatial device, not closely tied to the visual input domain (Baddeley, 1986). Also, Baddeley and Lieberman (1980) observed that performance in a visualization task (the Brooks matrix task) was disrupted by an auditory spatial secondary task (pointing at a pendulum on the basis of auditory feedback while blindfolded). A nonspatial visual secondary task - a brightness judgment - had no effects on performance, even though the input was visual. A similar result was reported by Smyth and Scholey (1994), who demonstrated in a number of experiments that an auditory spatial secondary task impaired a visual spatial main task (a Corsi test). These results support the notion that at some level, auditory and visual inputs share a component relevant for the maintenance of spatial information in working memory. It has been suggested that this component is spatial attention (Parmentier \& Jones, 2000; Smyth \& Scholey, 1994; Zimmer \& Speiser, 2002). During retention, spatial attention is oriented toward the to-be-remembered locations (Awh \& Jonides, 2001). If it is reoriented in space (e.g., to the location of the auditory signal of the secondary task), the shift disrupts the maintenance process. Among others, Merat and Groeger (2003; Merat, Groeger, \& Withington, 1999), who also reported interference between auditory spatial perception and visual spatial working memory, did not restrict interference to spatial attention, but assumed the common demand on general attentional resources to be causative (see also Klauer \& Stegmaier, 1997). This approach, however, leaves open the question of how auditory spatial information is maintained. 
All of the aforementioned experiments investigated the influence of auditory spatial secondary tasks on visual spatial main tasks. None of the studies directly compared memory processes in auditory spatial and visual spatial main tasks. Whenever spatial memory was investigated, auditory stimuli served as input to the secondary task, which required no retention of the auditory spatial information. Studies that did directly focus on interference between similar auditory and visual processes were concerned with attention and perception, not with memory. However, demonstrating that spatial attention has a supramodal component that allows for cross-modal interference (Driver \& Spence, 1998; Kennett, Eimer, Spence, \& Driver, 2001) does not say anything about maintenance of auditory spatial information. Therefore, the question of how auditory spatial main tasks are solved in working memory, in comparison with visual tasks, remains unresolved, as does the question of whether the VSSP might also be involved in auditory spatial memory tasks.

In its recent formulation, the VSSP is strongly associated with visual input. It stores visual features, spatial configurations, visual images, or the results of visual construction tasks (Logie, 2003; Logie \& Pearson, 1997; Quinn \& McConnell, 1999). Support for the strong relationship between the visual input domain and the VSSP is seen in the finding that visual imagery main tasks (e.g., the peg word mnemonic task) were impaired by additional visual input, even if the additional material is irrelevant and should be ignored by participants. In the secondary task conditions, participants either looked at irrelevant line drawings (Logie, 1986; Logie \& Marchetti, 1991) or viewed dynamic visual noise (i.e., changing patterns in a randomly filled black-and-white matrix; Quinn \& McConnell, 1999). It seems that irrelevant visual material overwrites the information held in the store, in a way similar to the irrelevant speech effect observed within the phonological loop (Salamé \& Baddeley, 1982). According to this position, the VSSP holds only visual information; hence, spatial information from auditory input cannot be directly represented within this mechanism.

Consequently, the initial processing of auditory spatial information should occur in some other subsystem. In principle, the perceptual subsystem could also be used for the short-term retention of sounds' locations, so the information would not have to be integrated with visual spatial information until a common representation of space was needed. For instance, locations could be encoded as part of the modality-specific object information-for example, alongside the colors of a picture or the frequencies of a tone - and thus be embedded in their specific modalities (i.e., the visual modality for pictorial input and the auditory for spatially distributed sounds). Then, two independent modality-specific short-term memory systems would be used: The visual cache for visual input, and some kind of auditory cache for holding spatial information in the format in which it was perceived (i.e., coded as temporal and frequency features of the sound).

Another possibility remains, of course. Auditory spatial information might be recoded into the format of visual images within VSSP, after the auditory objects have been recognized within that modality-specific system. Auditory spatial information would then only be used to build up a visual spatial representation by means of mental imagery. Such recoding could, for example, be accomplished pictorially by including more or less detailed visual imagery of the heard objects in their respective positions.

We can summarize that perception of spatial positions seems to occur in a modality-specific way-that is, by extracting the spatial properties of sounds and images, respectively. This seems to be the case despite an amodal component in spatial attention, which is also engaged in visual spatial rehearsal. From studies on working memory, it seems that the information subjected to visual spatial short-term retention is not abstract, but similar to the perceptual format of a retinotopic image. Auditory spatial information should therefore either be represented in its own, perception-like system, which could be considered an independent auditory spatial working memory, or be recoded into the VSSP by way of visual imagery.

Besides the possibilities just mentioned, a further line of research has to be discussed: the distinction between a dorsal where and a ventral what path (Ungerleider \& Mishkin, 1982) in the context of spatial memory. There is evidence that different types of information - about what is seen and where it is seen-are processed via different neural pathways (for a review, see Goodale \& Humphrey, 1998). Visual object information is processed in the ventral pathway from occipital to inferior temporal cortex, and visual spatial information activates the dorsal stream from occipital to inferior parietal cortex. The question why such a distinction is made by the brain has led to the notion that perception has to fulfill different functions (Goodale \& Milner, 1992). Whereas spatial perception is needed for directed movements, and thus needs to activate motor behavior as fast as possible, object information is needed for identification, and is therefore optimized to activate past knowledge. Hence, the visual system is twofold in terms of subsequent processing. This logic cannot simply be transferred from visual perception to visual working memory, but the perceptual division should nevertheless influence memory, if it holds true that working memory recruits posterior cortical systems already involved in the perceptual processing of the same material (Ruchkin, Grafman, Cameron, \& Berndt, 2003). Accordingly, the anatomically distinct processing of what and where information has proven relevant for visual spatial short-term memory (Bosch, Mecklinger, \& Friederici, 2001; Mecklinger \& Pfeifer, 1996; E. E. Smith et al., 1995; Ungerleider, Courtney, \& Haxby, 1998), as well as for mental imagery (Mazard, Tzourio-Mazoyer, Crivello, Mazoyer, \& Mellet, 2004).

When it comes to auditory processing, the division is less obvious. Early visual processing is retinotopic, and thus spatial from the outset. In contrast, early auditory processing is organized along frequency, and evidence for a cortical space map is lacking (Middlebrooks, 2002). The anatomical segregation into ventral and dorsal pathways has also been found for auditory perception (Arnott, Binns, Grady, \& Alain, 2004; Bushara et al., 1999; Herrmann, 
Senkowski, Maess, \& Friederici, 2002)—with both paths leading from Wernicke's to Broca's area (Parker et al., 2005) - but their function is less clear. Some authors suggest that the division serves speech perception and allows for the distinction between speaker and message (Belin \& Zatorre, 2000). The ventral pathway processes information relevant for the identification of the sound source (timbre, speaker, or instrument), and the dorsal pathway processes other information that serves to identify the sound event (pitch, sentence, or melody; see Z. M. Smith, Delgutte, \& Oxenham, 2002). Thus, the auditory ventral pathway seems to serve object identification, as in the visual case, whereas the dorsal pathway does not process purely spatial information, but instead frequency information that, among other things, allows for spatial localization in further processing.

As a consequence of the distinction between what and where in visual processing, positional memory per se (without object information) has been distinguished from memory for the conjunction of object and position (see, e.g., Postma \& de Haan, 1996). Support for this notion can be found in patients showing a double dissociation between memory for locations per se and object-location binding (Kessels, de Haan, Kappelle, \& Postma, 2001; Kessels, Kappelle, de Haan, \& Postma, 2002). The information concerning object-location binding in turn might be held in temporary representations of perceived objects to which spatial information is attached. Candidates for this binding function are the perceptual object files introduced by Treisman (for reviews, see Treisman, 1998, 2006).

In accordance with this approach, Schneider (1999) suggested that object files also constitute visual short-term memory (see also Wheeler \& Treisman, 2002). The episodic buffer (Baddeley, 2000) could be seen as an equivalent to this conception within the multicomponent working memory model (see also Zimmer, Speiser, \& Seidler, 2003). If one follows this line of thinking, neither a visual nor an auditory spatial buffer would be used to remember where an object or a sound was located, but object files would be used instead. Although object files collect information from specific perceptual modalities, they are in principle supramodal and may represent information from different modalities, and may also include pointers to longterm memory entries. In this case, object files generated from auditory and visual input would coexist in a supramodal working memory. According to this view, working memory is constituted by object files representing environmental objects perceived in any modality. These representations can be thought of as links between the modalityspecific items and a common ego-centered representation of space. Because these files are supramodal, both modalities share the same spatial working memory.

In summary, there are two main possibilities for the relationship between auditory and visual working memory of spatial locations. First, location information could be encoded and stored in separate modality-specific compartments: the visual cache proposed by Logie (1995) for maintaining locations of seen objects, and a corresponding auditory cache maintaining location information of heard sounds. The spatial properties of the input would then be upheld in the low-level format in which they are originally perceived - as retinotopic spatial distributions in the case of pictures and as tonotopic representations of temporal and frequency features in the case of sounds.

Second, object location information could be stored in a common working memory. This may be a supramodal representation, as suggested in the conception of object files. Alternatively, the visual cache could be dominant for location information, storing not only visual objects and their positions, but also location information from any modality by means of recoding into a visual layout.

In order to find out how auditory spatial information is maintained in working memory relative to visual spatial information, the current study explored to what extent aurally and visually presented object locations draw on the same or different memory resources. For this purpose, we made use of the fact that working memory resources are limited; increasing memory load reduces memory performance. This logic can be used to test the multiple-spatial working memory hypothesis, if we make the plausible assumption that each system has its own capacity. If the load can be distributed over different memory systems, memory performance for two-modality input should be higher than for single-modality input. For that reason, we compared memory performance using pure-modality lists (either $n$ pictures or $n$ sounds) versus mixed lists (containing items from both modalities-i.e., $n / 2$ pictures and $n / 2$ sounds). If modality-specific memory systems contribute to object location memory, both resources should be combined for the retention of mixed-modality lists. Because each working memory system has to retain only $n / 2$ items, memory performance in the mixed-list case should be higher than in the pure-modality case, in which one system has to hold all $n$ items. Differences should at least show up when the respective resource reaches its capacity limit. We therefore also introduced a total memory load manipulation $(4,6$, or 8 items). If there are indeed independent resources for auditory and visual items, performance for mixed lists should be comparable to that for pure lists of half the length.

Penney (1989) reviewed studies of verbal short-term memory that used a similar research strategy, by presenting either mixed lists of written and spoken words or lists containing items in only one modality. Memory was improved when items were presented both aurally and visually, in comparison with the pure-modality condition. She concluded that verbal short-term memory includes not only a phonological code, but also modality-specific contributions of visual and auditory codes.

Following a similar experimental logic, we seek to find the amounts of modality-specific contributions to spatial working memory.

\section{EXPERIMENT 1}

\section{Method}

Participants. Twenty-four students of different departments at Saarland University participated in the experiment for payment ( $€ 8$ for approximately $1 \mathrm{~h}$ ). 
Materials and Design. The stimuli consisted of 40 pictures and 40 sounds with matched object identity; that is, 40 different objects were available in both modalities. Thus, only objects that produce sounds were used-musical instruments, electric appliances, machines, and animals. ${ }^{1}$ The visual stimulus material consisted of 40 colored drawings of these objects. The auditory material consisted of the respective sounds these objects make, played for $2 \mathrm{sec}$ each. Hence, the lists were identical with regard to semantic content.

In order to realize a spatial working memory task, we presented these objects at specific locations in the environment, and these locations were the same for seen and heard objects. For that purpose, we installed a large silver screen about $2.5 \mathrm{~m}$ wide (azimuth $\sim 50^{\circ}$ ). Four horizontally aligned locations were defined as study positions (azimuth deviation from central position about $-23^{\circ},-8^{\circ}, 8^{\circ}$, and $23^{\circ}$, respectively). A fifth position $\left(0^{\circ}\right)$ in front of the participant was used to present the test items. Visual items were projected onto these positions on the screen; auditory items were presented by loudspeakers placed behind the screen at each of the used positions.

We allocated the to-be-remembered material to lists of three different set sizes (four, six, and eight items). This manipulation defined the variable memory set. These memory sets of four, six, or eight objects were presented as pictures and sounds - either in pure-modality or mixed-modality sets. The modality manipulation (pure visual, pure auditory, and mixed modality) defined the variable list modality. In mixed-modality sets, half of the objects were presented as pictures and the other half as sounds. Items were randomly drawn from the pool of 40 objects in a concept-wise fashion. In other words, both the sound (e.g., a bark) and the image (e.g., a $\mathrm{dog}$ ) referring to the same concept were drawn in parallel, but only one was presented, either the sound or the image. Thus, a specific object was never presented in both modalities within one memory set. When all objects had been drawn, the item pool was reinitialized. The location for each item was randomly drawn from the four possible locations without replacement; after four draws, the set was reinitialized. Hence, with set sizes larger than four, two objects were presented at some locations. Altogether, the participants processed 8 lists of each set size and modality, for a total of 72 lists.

At test, two items were selected from each list. The first test item always was selected from the first half of the list, and the second from the second half. Items from all memory set positions were tested equiprobably. For mixed-modality sets, the two selected test items could both be visual, both be auditory, or modalities could be mixed.

The variables memory set and list modality varied within subjects. Set size was held constant within blocks, whereas modality varied randomly.

Procedure. The participants studied sequentially presented lists of different lengths, and after a short interval they were given two pseudorandomly selected items from the list. The test items were presented sequentially on a central screen position not used for study. The participants were instructed to remember the positions of sounds and pictures and later, during test, to assign the test stimuli to the positions they had occupied in the study phase. To indicate positions, the participants used a custom-made keyboard with four keys arranged in the same linear configuration as the four possible study positions.

Each participant was seated $2.5 \mathrm{~m}$ away from the screen. The study items were sequentially presented for $2 \mathrm{sec}$ each, with an interstimulus interval of $1 \mathrm{sec}$, resulting in a study phase of 11,17 , or $23 \mathrm{sec}$ (for four, six, and eight items, respectively). After a retention interval of $3.5 \mathrm{sec}$, the first test stimulus was displayed for a maximal time of $2 \mathrm{sec}$. The participants indicated its position by a keypress, which terminated the item presentation. If no response was given during item presentation, a pause followed after stimulus offset, until an answer was given (maximally $5 \mathrm{sec}$ ). The second test stimulus followed $1 \mathrm{sec}$ after the keypress, and $2.5 \mathrm{sec}$ after the last response, a new trial started. In order to prevent the participants from confusing study and test phases, the words Bitte zuordnen ("Please assign") were displayed at the top of the screen $500 \mathrm{msec}$ before the presentation of a test item, and the words Neue Lernphase ("New study phase") were displayed $2 \mathrm{sec}$ before a new trial started.

Memory set size was manipulated between blocks. The first block consisted of lists of size four, the second of size six, and the third of size eight. The blocks were presented in ascending set sizes to allow the participants to get accustomed to the task before increasing its difficulty. Before starting a new block, they were informed of the new set size and had the opportunity to pause. Modality always varied randomly within each block. Hence, the participants could not predict the modality of the upcoming items.

\section{Results and Discussion}

The data were analyzed on an item basis, so that items of mixed lists were differentiated by modality, resulting in the two variables list type (pure and mixed) and item modality (auditory and visual).

A $3 \times 2 \times 2$ ANOVA with the variables memory set, list type, and item modality was conducted on the proportions of correctly relocated items (hits). Mean hits as a function of the three variables are presented in Figure 1. For all variables with more than two levels, degrees of freedom were corrected for nonsphericity (Greenhouse $\&$ Geisser, 1959). In these cases, original degrees of freedom, corrected $p$ values, and the correction coefficient $\varepsilon$ are reported; this practice was analogously applied in all other experiments as well.

The analysis yielded significant main effects for all three variables. Hits declined with increasing memory set $\left[F(2,46)=82.43, M S_{\mathrm{e}}=0.0142, p<.0001, \varepsilon=.94\right]$. Performance was highest with four-item sets (.83), decreased with six items (.68), and was lowest with eight items (.61). This main effect was exhaustively explained by a linear trend $\left[F(1,23)=147.0, M S_{\mathrm{e}}=0.0153, p<\right.$ $.0001]$, with no significant residual. In addition, there was an effect of list type, which showed an advantage for mixed (.73) over pure (.69) lists $\left[F(1,23)=15.28, M S_{\mathrm{e}}=\right.$ $0.0086, p<.001]$. Relocalization performance for auditory items (.62) was worse than for visual items (.80) $\left[F(1,23)=94.76, M S_{\mathrm{e}}=0.0233, p<.0001\right]$. The three variables did not interact significantly.

As can be seen in Figure 1, performance in mixed and pure lists was highly similar, except for sounds in the fouritem condition. Presumably, the main effect of the variable list type is due to this difference. We therefore did pairwise post hoc comparisons (Fisher's LSD test) of mixed versus pure lists for each set size and modality. In the auditory four-item condition only, location memory clearly differed for items from mixed versus pure lists $(p<.0001)$. For all other combinations of set size and modality, scores from both list types did not differ.

The results were clear cut. Location memory decreased with memory set size and was better for pictures than for sounds, but there was no benefit caused by mixed modalities. The result that location memory is lower for sounds than for pictures has already been observed in some studies (see, e.g., Zimmer \& de Vega, 1996) and may be due either to differences in the spatial resolution of the two systems or, relatedly, to the ease of extracting spatial information from their respective perceptual inputs. Visual 


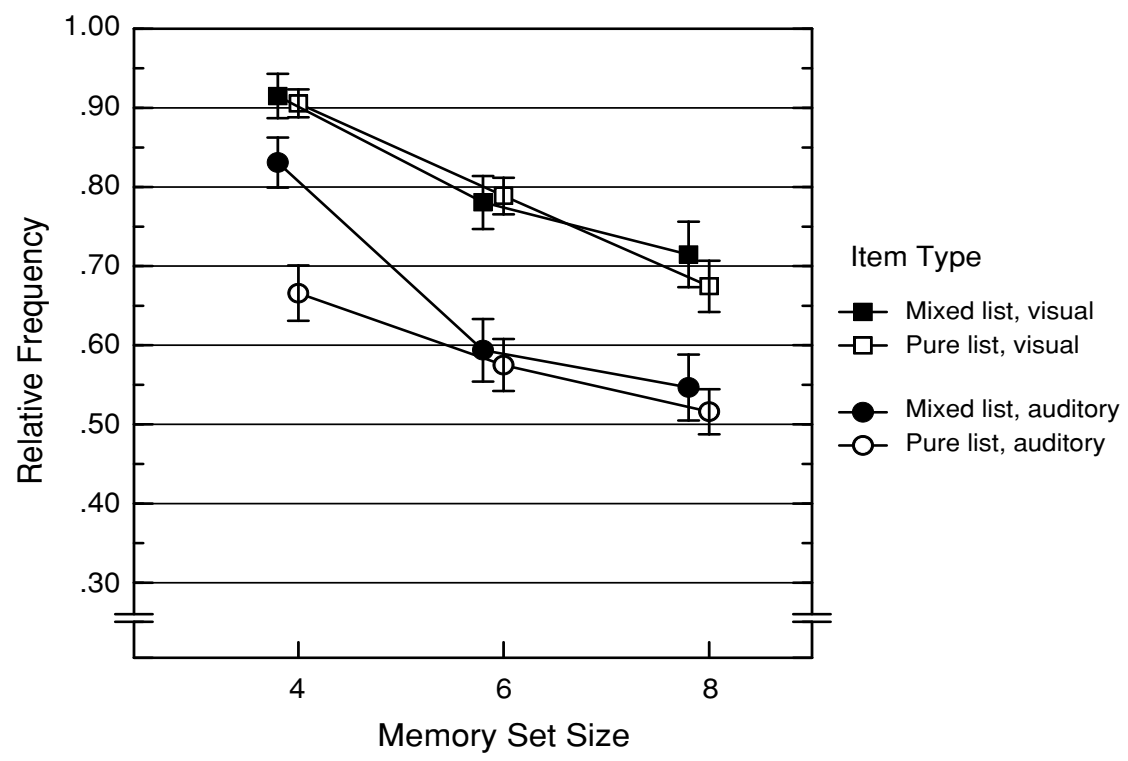

Figure 1. Experiment 1: Relative frequencies of hits by item type and memory set, including means and standard errors.

input may lead to more accurate spatial representations than does auditory input, an idea supported by the results of visual imagery research (for a review, see Paivio, 1986). The quality of input in the two systems is inherently different to begin with.

In our experiments, the performance difference between auditory and visual items is most likely due to perceptual processes, since it not only showed up in memory tasks, but also in a simple localization task performed in the same setting: We asked participants to indicate directly the positions of visual and auditory stimuli during presentation and observed performance differences for the different modalities. Presented in the identical spatial layout, visual items could be located better (hits .97, $S D .01$ ) than could auditory stimuli (hits .82, $S D$.03) $[t(16)=7.58$, $p<.0001]$.

The memory load manipulation yielded the expected effect of memory performance declining with larger set size. This memory load effect was the same in both modalities, suggesting that spatial information in both modalities is represented in a similar way. This conclusion is supported by the fact that performance did not benefit from the mixed presentation. The locations of only three sounds in the context of three pictures (i.e., six items in total) were not remembered better than the locations of six sounds in a pure list, and performance was worse than it would have been for only three sounds alone (as estimated from the four-item list). The same was true for pictures. According to the logic of limited resources, these results can be taken as support for the assumption of a common memory system contributing to temporary location memory for sounds and pictures. If two separate mechanisms were available for visual and auditory materials, each having its own resources, items would be distributed according to their modality in the mixed-list condition. Memory performance within one modality in the mixed-modality condition should thus equal memory performance in the respective pure-modality list with the same intramodality list length (i.e., half the total length). Performance for the four auditory items in the eight-item mixed list, for example, should be the same as performance for the auditory items of the four-item pure list, because both draw on auditory spatial memory to the same degree. This effect was not observed, however. We therefore assume that location memory for both sounds and pictures relies on the same process.

The exception to these results was the list length of four items. Locations of sounds from a mixed four-item list were remembered better than those from a pure four-item sound list. With pictures this effect was not observed, but we have to take into account that there was little room for such an improvement, because spatial memory for four pictures was nearly perfect. One might speculate that this result indicates a lower capacity of spatial working memory for sounds than for pictures. For the latter, four items has been suggested as the upper limit for storage (Luck \& Vogel, 1997). Thus, sounds from mixed-modality lists of four items might have had an advantage over those in single-modality lists because their two sounds did not exceed storage capacity. Location memory for longer lists might then be supported by supramodal long-term memory, and therefore would not show the mixed-list advantage. We do not, however, consider this possibility likely. First, if this were true, one would also expect an advantage for six-item mixed-modality lists over the singlemodality lists, because in this case only three sounds were presented. Furthermore, with a list length of six or eight pictures, which should exceed visual storage capacity, this effect should also occur. Second, there is a more plausible alternative explanation: namely, the reduction of spatial 
uncertainty, or — simply speaking — a guessing advantage. In a four-item list, every location was used exactly once, which was not the case with longer lists, and the locations of visual items from four-item sets were remembered almost perfectly. As a consequence, in mixed lists with four items the remembered positions of the visual items could be excluded as the locations of sounds, and thus only two positions remained for the relocation of the auditory items. This advantage did not exist for the longer lists, because in this case more than one item could be presented in each position. In Experiment 3, we directly addressed this notion of a reduction in spatial uncertainty for auditory items in the mixed four-item sets.

\section{EXPERIMENT 2}

The data of Experiment 1 suggest that temporary location memory for auditory and visual materials is not provided by modality-specific compartments, but by the same supra- or amodal process. However, although this conclusion is in line with the data, alternative explanations are possible. In the first experiment, we used sounds and pictures from the same semantic category-for example, a picture of a dog and the sound of barking. We did so to keep constant any influence of semantic memory on recall. Nonetheless, one could object that under these conditions, participants remembered the names of the items and their locations, instead of memorizing any modalityspecific information. Within the multicomponent working memory model, the supramodal episodic buffer (Baddeley, 2000) is thought to bind identity (verbal labels) and location information, so if participants did use verbal encoding, we might have missed a modality-specific buffer. In order to test this explanation, we conducted a second experiment with new stimulus materials that did not allow for easy naming.

To eliminate the possibility of a verbal recoding strategy, we presented meaningless pictures and sounds in Experiment 2. The pictures were random shapes, and the sounds were clearly distinct auditory events that could not be identified as anything specific. If temporary spatial location memory really is supramodal, the pattern of results obtained in Experiment 2 should be similar to that found in Experiment 1. If, on the other hand, the pattern in Experiment 1 resulted from a verbal recoding strategy, the pattern should change in this new experiment, because participants were hindered from using a verbal strategy to memorize the items.

\section{Method}

Participants. Twenty-four students from different departments at Saarland University participated in the experiment. They were paid $€ 8$ for a session of approximately $1 \mathrm{~h}$.

Materials. The stimuli used in Experiment 2 consisted of 40 pictures and 40 sounds that did not constitute identifiable objects. The visual stimulus material consisted of 40 multicolored meaningless figures, each assembled from several geometric shapes. The sounds, played for $2 \mathrm{sec}$ apiece, were composites of several short, machinelike noises (like creaking, clattering, squeaking, and bubbling; the components were drawn from a pool of 50 sounds and were assembled in different temporal patterns).

Design and Procedure. The design and procedure were the same as in Experiment 1 . The variables memory set (four, six, and eight items) and list modality (pure visual, pure auditory, and mixed modality) were varied within subjects.

\section{Results and Discussion}

The data were analyzed in a $3 \times 2 \times 2$ ANOVA with the variables memory set (four, six, and eight items), list type (pure and mixed), and item modality (auditory and visual). Mean hits as a function of the three variables are presented in Figure 2.

All three variables and the three-way interaction yielded significant effects. With increasing memory set size, the proportion of hits decreased across four (.62) to six (.52) to eight $(.46)$ items $\left[F(2,46)=22.25, M S_{\mathrm{e}}=0.0307\right.$, $p<.0001, \varepsilon=.84]$. Again, there was a clear linear trend $\left[F(1,23)=30.66, M S_{\mathrm{e}}=0.0439, p<.0001\right]$, without residual. Items from pure (.50) and mixed (.57) lists differed significantly $\left[F(1,23)=16.93, M S_{\mathrm{e}}=0.0228, p<\right.$ $.0005]$, and relocation performance was better for pictures (.64) than for sounds $(.42)\left[F(1,23)=89.97, M S_{\mathrm{e}}=\right.$ $0.0388, p<.0001]$.

In addition, the three-way interaction was significant $\left[F(2,46)=4.42, M S_{\mathrm{e}}=0.0247, p<.05, \varepsilon=1.0\right]$. It was examined through pairwise post hoc comparisons (Fisher's LSD test) of mixed versus pure lists for each combination of set size and modality, which revealed a significant difference for auditory four-item lists of pure versus mixed modality $(p<.0001)$. In all other cases, scores did not differ with regard to list type.

In Experiment 2, the unusual stimulus materials led to lower overall performance but did not change the general pattern of the results. Thus, there is no evidence that the pattern in Experiment 1 resulted from a verbal strategy suggested by the familiar and easily nameable stimulus materials. If verbal strategies were responsible for the observed pattern in both experiments, in Experiment 2 the load manipulation should have led to a larger decline, for any naming difficulties should have exerted a progressively stronger influence in sets with increasing numbers of items. If naming is difficult, it should become even more difficult as there is more material to be named, leading to a multiplicative effect of the load and material manipulations. In a conjoint analysis of the two experiments, with the between-experiments variable stimulus material, the familiar stimuli of Experiment 1 led to better overall performance (.71) than did the nonsense stimuli (.53) $\left[F(1,46)=29.57, M S_{\mathrm{e}}=0.1503, p<.0001\right]$. However, the stimulus material variable was not involved in significant interactions.

Although we conclude that memory for spatial positions was not achieved by verbal rehearsal, we do think that conceptual information plays a crucial role in discriminating the individual items; hence, the better performance in Experiment 1. The items in Experiment 2 could not activate sufficiently distinct long-term memory entries, 


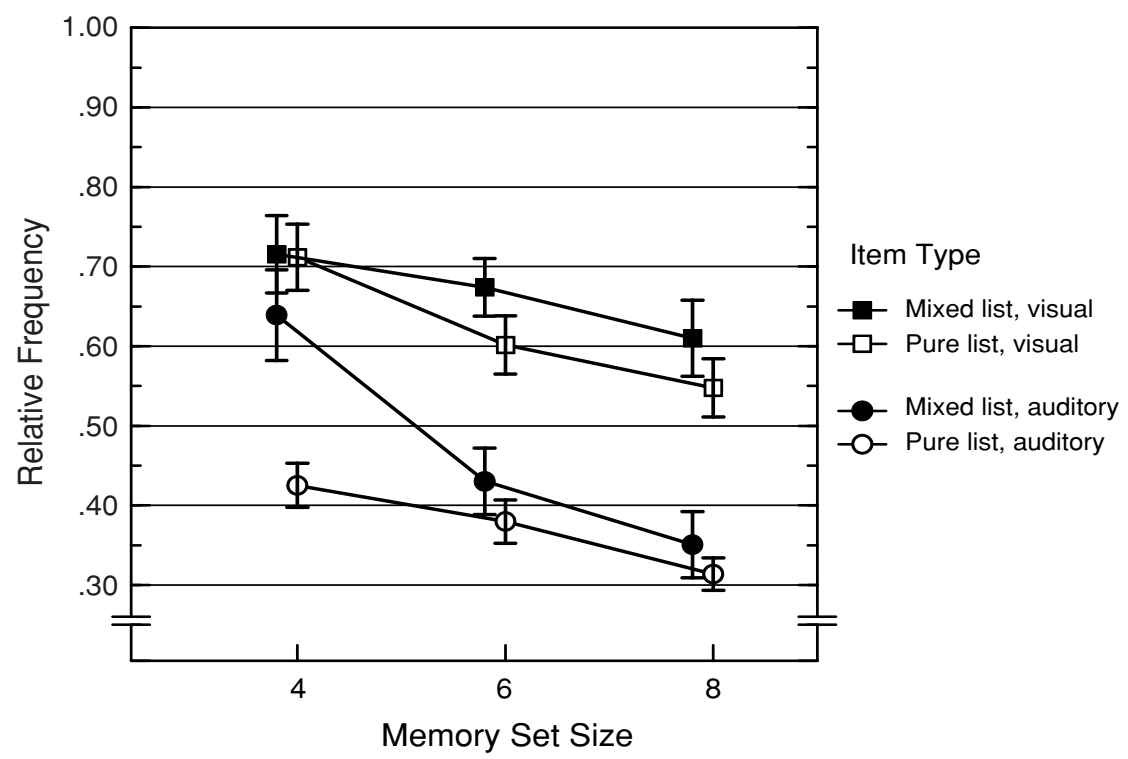

Figure 2. Experiment 2: Relative frequencies of hits by item type and memory set, including means and standard errors.

and thus were more often confused and assigned to wrong positions as a result. On that account, the performance difference between the two experiments could mirror differences in the ability to discriminate list items - which is a necessary prerequisite for remembering their specific locations - but not in position memory per se. ${ }^{2}$ The slight advantage of mixed lists in Experiment 2 may similarly have stemmed from the use of item modality as a criterion for discriminating list items, in absence of better conceptual information. Participants may have remembered whether an auditory or a visual item had appeared on a particular position, yet without remembering the actual object-to-position mapping.

The unfamiliar stimulus materials of Experiment 2with its reduced semantic information - not only prevented easy naming of the stimuli, but also prevented auditory items from being recoded into a picture-like representation. Unlike the familiar sounds in Experiment 1, those in Experiment 2 did not refer to visual objects. Though the results of both experiments provide evidence for a common resource for auditory and visual location information, it is unlikely that this resource is the verbal rehearsal mechanism or a pictorial representation in VSSP.

The results of Experiment 2 also confirmed that the pattern of results from Experiment 1 persists with a lower level of overall performance. With four-item lists, there was again an advantage for auditory items from mixedmodality rather than pure-modality lists, just as we had observed in Experiment 1. However, in the previous experiment, performance with visual material was so high that we could not exclude a ceiling effect as a cause for the absence of the same effect with visual items. Experiment 2 demonstrated that the advantage really is confined to auditory material, because memory performance in both visual conditions was substantially lower than before. Experiment 3 was designed to test whether our explanation given above is correct.

\section{EXPERIMENT 3}

We hypothesized that location memory for auditory items in mixed-modality four-item lists benefited from the very good memory performance of their visual context items. When the locations of visual items were remembered almost perfectly, only two positions were left for the auditory items. Thus, spatial uncertainty for the auditory items in mixed lists was significantly reduced relative to pure lists. Assuming that this is correct, the mixedmodality advantage for four-item lists should disappear if spatial uncertainty is the same in mixed-modality and pure-modality lists. To test this, we manipulated spatial uncertainty by varying the number of possible study and test locations of four-item lists.

In the invariant condition, we presented items in a way that made spatial uncertainty independent of list structure. We used only two study positions, while retaining all four test positions. In this case, with a four-item list, positions were always used twice, so that spatial uncertainty in the mixed-modality case was the same as in the pure-modality case. In this situation, with mixed lists, remembering where a visual item was presented did not help localize where sounds had been presented. Hence, the advantage of the mixed-modality list should disappear if spatial uncertainty - and not modality-dependent resource capacities - was the origin of the mixed-modality effect.

In the standard condition, we used the same study-test procedure as before, so that spatial uncertainty differed with list type. Here we expected an advantage of the 
mixed compared with the pure list condition, replicating the previously observed results.

In the reduced condition, we tried to positively show that reduced spatial uncertainty also enhances memory in pure-modality lists. To this end, we used four study positions, but crossed out two false response alternatives during test, simulating the exclusion of response options due to remembered visual item positions. Consequently, we expected to find in pure-modality lists a memory advantage comparable to the one resulting from the influence of visual context items on auditory items in mixed-modality lists. Memory performance should be enhanced compared with the standard condition.

\section{Method}

Participants. Seventeen students of different departments at Saarland University participated in the experiment. They were paid $€ 8$ per hour.

Materials. In Experiment 3, we used the same 40 identifiable sounds and pictures used in Experiment 1.

Design and Procedure. Only memory sets of four items were used. Spatial uncertainty was manipulated by varying the number of study and test positions. Each of the three conditions was presented on one third of the trials. In the invariant-uncertainty condition, two positions were each used twice at study, but all four locations were available as test alternatives. In the standard condition, the four items were presented in four different study positions, each used once, and all four locations were available as test alternatives. In the reduceduncertainty condition, all four locations were used at study, but two locations were crossed out during test by a red $X$, indicating that those positions were not valid locations for that item. The $X$ was presented from $500 \mathrm{msec}$ before stimulus onset until its disappearance. Except for these changes, the experimental procedure remained the same as in Experiment 1.

Thus, we had two variables that varied randomly within subjects: spatial uncertainty (invariant, standard, and reduced) and list modality (pure visual, pure auditory, and mixed). In order to match the number of items per modality in the mixed-modality lists to that in the pure-modality lists, we doubled the number of mixed-modality trials to equal the combined number of visual and auditory trials.

\section{Results and Discussion}

Mean hits are presented in Table 1 . A $3 \times 2 \times 2$ ANOVA with the variables spatial uncertainty (invariant, standard, and reduced), list type (pure and mixed), and item modality (auditory and visual) yielded significant main effects for spatial uncertainty $\left[F(2,32)=4.40, M S_{\mathrm{e}}=0.0147\right.$, $p<.05, \varepsilon=.96]$, and item modality $[F(1,16)=32.65$, $\left.M S_{\mathrm{e}}=0.0329, p<.0001\right]$. There also was a significant interaction between the two variables $[F(2,32)=5.62$,

Table 1

Experiment 3: Relative Frequency of Hits by Item Type and Spatial Uncertainty

\begin{tabular}{|c|c|c|c|c|c|c|}
\hline \multirow[b]{2}{*}{ Item Type } & \multicolumn{2}{|c|}{ Invariant } & \multicolumn{2}{|c|}{ Standard } & \multicolumn{2}{|c|}{ Reduced } \\
\hline & $M$ & $S E$ & $M$ & $S E$ & $M$ & $S E$ \\
\hline \multicolumn{7}{|l|}{ Auditory } \\
\hline Pure & .74 & .03 & .72 & .04 & .79 & .04 \\
\hline Mixed & .72 & .04 & .80 & .04 & .88 & .03 \\
\hline \multicolumn{7}{|l|}{ Visual } \\
\hline Pure & .93 & .02 & .89 & .03 & .93 & .02 \\
\hline Mixed & .92 & .03 & .92 & .02 & .93 & .02 \\
\hline
\end{tabular}

$\left.M S_{\mathrm{e}}=0.0075, p<.05, \varepsilon=.70\right]$. List type showed no significant main effect or interaction.

A post hoc comparison (Fisher's LSD test) was conducted to compare items from mixed versus pure lists for each modality and uncertainty condition. It confirmed that the means for visual items in all conditions did not deviate from one another (all $p \mathrm{~s}>.50$ ). As predicted, there was no advantage for auditory items from mixed lists over those from pure-modality lists in the invariant-uncertainty condition (.74 vs. .72; $p>.70$ ). However, a marginally significant mixed-modality advantage was observed in those conditions in which remembering visual item positions could be used to reduce spatial uncertainty - the standard condition $(.72$ vs. $.80 ; p<.06)$ and the reduceduncertainty condition $(.79$ vs. $.88 ; p<.06)$.

The important result is that with invariant spatial uncertainty, relocation performance was the same for both auditory conditions (i.e., items in pure- and mixed-modality lists) and was similar to performance for pure-list items in the standard condition. These results support the hypothesis that auditory items from mixed lists were not remembered better, but benefited from a reduction in spatial uncertainty. With four items and each position used once, remembering the locations of the two visual items left only two positions for the auditory items, and this enhanced guessing by means of partial knowledge.

In the reduced-uncertainty condition, we simulated this effect of the visual context items by reducing the number of test positions. Crossing out two positions at test had the same effect on pure-list auditory items as remembering the visual item positions in the mixed-list condition. Indeed, location memory for pure-list auditory items (proportion correct .79) now reached the same level as location memory in mixed lists with the standard procedure $(.80){ }^{3}$

Taken together, the results of Experiment 3 strongly support the hypothesis that better performance with auditory items from four-item mixed lists, as observed in Experiments 1 and 2 and the standard condition of Experiment 3 , is due to a reduction in spatial uncertainty resulting from an interdependency of study positions, not to the distribution of items to separate modality-specific mechanisms, each having its own capacity.

\section{GENERAL DISCUSSION}

The aim of the present research was to test whether separate working memories for spatial information exist for auditory and visual spatial input. A candidate for the storage of visual spatial information is the visual cache (Logie, 1995), and analogously an auditory cache might store the direction in which a sound source is located. If such an auditory cache existed, humans would have available two independent part-systems for the storage of spatial information provided by visual and auditory input, respectively. Alternatively, one could suggest that the storage of spatial object information is unitary, whether it is provided by a seen picture or a heard sound. Object files (see Treisman, 1998) would be suitable for that function, 
because they integrate or bind sensory-specific features of the represented object, its identity, and its location. Schneider (1999) proposed that visual spatial working memory contains up to four object files (see Luck \& Vogel, 1997) - that is, temporary episodic representations of objects, containing high-level attributes such as shape, color, and location bound together by an object index. Although his model has only been discussed as a visual spatial workspace, it could easily be extended to auditory spatial memory.

In order to decide between modality-specific and unitary storage, we presented sounds and pictures in pureand mixed-modality sets and tested short-term memory for their locations. If distinct memories for the locations of auditory and visual objects existed, each having a specific capacity, performance decline with increasing set size should differ depending on each store's capacity limit. Furthermore, mixed-modality lists should then lead to better memory performance than pure-modality lists, because memory load would be distributed to the two systems. In contrast, if a common location memory for items of either modality existed, we should observe no advantage in the mixed-modality condition. The results of all three experiments supported the view of a unitary location memory: The decrease in memory performance with increasing set size was the same for both modalities, and mixed-modality lists did not show any advantage over pure-modality lists.

Our results constitute evidence that locations of auditory and visual input are stored in a common memory. Furthermore, it was shown that the common format is not the phonological code provided by the names of the items. Although the results were qualitatively the same for items that could be named (Experiment 1) and items that could not be named (Experiment 2), memory performance for the arbitrary items was lower than for the meaningful items. The latter result had already been observed using a task in which configurations of pictures were to be reconstructed (Zimmer, Speiser, \& Seidler, 2003), suggesting that the meaning of the items also plays a role in temporary spatial memory, and hence that location is not held in a presemantic store.

Within the multicomponent working memory model, the episodic buffer serves the purpose of maintaining multimodal information in a common store (Baddeley, 2002). It is a short-term store that contains entries binding different types of information. The episodic buffer could therefore be thought of as a device retaining the link between an object and its location, regardless of the modality in which the object and location information are presented. The episodic buffer is capable of binding information provided by domain-specific long-term memory structures, and it temporarily maintains these entries, keeping them available in working memory. The suggestion of an episodic buffer is in this respect comparable to the proposal that working memory builds new episodic links between activated long-term memory representations (Cowan, 1999, 2001). Because the idea of bound episodic information is already contained in the concept of object files, we use the term object file for active memory entries. These units bind all information belonging to an object: information on the identity of an item, which includes a modality-specific representation of sensory object features; information on its spatial location; and probably also information relevant for the prevailing action and the current task (see Hommel, 1998).

Object files bind information across different modalities, and this creates the possibility that the visual cache is still involved in temporary location memory, even if input is auditory. Spatial information might be held in visual spatial working memory (see Postma \& de Haan, 1996) and might then be linked to nonspatial object information by object files. According to this position, spatial representations are peculiar to the visual subsystem, so that spatial information provided by any other modality is recoded as a location within the visual cache. As a consequence, temporary spatial memory of different modalities would be provided by the same mechanism, but different part-systems would be involved in memorizing the stimulus information as a whole.

The latter possibility offers the option that the visual cache of visual spatial working memory nevertheless serves as a unitary system representing location information in all cases. Participants might have visualized the spatial layouts, generating images of the sound objects. We do not consider this relevant in the type of task we have realized. Experiment 2 should have rendered visual imagery of the auditory events impossible, since the machine-like sounds did not correspond to imaginable visual objects. In a further series of experiments, we tested the contribution of modality-specific information to spatial short-term memory by introducing nonspatial secondary tasks that drew on the same memory resources as the spatial main tasks. We orthogonally combined visual and auditory secondary tasks with visual and auditory spatial main tasks. We observed general interference effects, but no modality-specific effects and no hint of a specific contribution of the visual component to spatial memory (Zimmer, 2006).

We therefore believe that in the type of task we have realized-memory for locations in a person's spatial environment - information from the visual cache is not used. Rather, we think object files represent location information by referring to a common representation of the egocentric environmental space shared by all modalities. For each attended item perceived in any modality, an object file exists that can be considered to represent episodic working memory. The object files are transient units consisting of active entries in domain-specific longterm memory structures, plus an additional component that temporarily binds these structures as long as the object file is active. This additional component might be attention (Treisman, 2006). Each file binds a multitude of heterogeneous information, only part of which is provided by modality-specific structures. When memorizing an auditory item location, for instance, the bound memory content would consist of sound information, location information, and some conceptual information (to the extent that 
the sound could be identified). In the case of a seen object, visual perceptual information would be represented rather than sound information. As a consequence, working memory is a net of active components distributed over several part-systems, some of which are modality specific, others shared across modalities (Ruchkin et al., 2003).

\section{REFERENCES}

Arnott, S. R., Binns, M. A., Grady, C. L., \& Alain, C. (2004). Assessing the auditory dual-pathway model in humans. NeuroImage, 22, 401-408.

AwH, E., \& JoNIDES, J. (2001). Overlapping mechanisms of attention and spatial working memory. Trends in Cognitive Sciences, 5, 119-126.

BADDEley, A. [D.] (1986). Working memory. Oxford: Oxford University Press.

BAdDeley, A. [D.] (2000). The episodic buffer: A new component of working memory? Trends in Cognitive Sciences, 4, 417-423.

BADDELEY, A. D. (2002). Is working memory still working? European Psychologist, 7, 85-97.

Baddeley, A. D., \& Hitch, G. (1974). Working memory. In G. H. Bower (Ed.), The psychology of learning and motivation (Vol. 8, pp. 647-667). Hillsdale, NJ: Erlbaum.

Baddeley, A. D., \& Lieberman, K. (1980). Spatial working memory. In R. S. Nickerson (Ed.), Attention and performance VIII (pp. 521-539). Hillsdale, NJ: Erlbaum.

Baddeley, A. D., \& Logie, R. H. (1999). Working memory: The multiple-component model. In A. Miyake \& P. Shah (Eds.), Models of working memory (pp. 28-61). Cambridge: Cambridge University Press.

Belin, P., \& Zatorre, R. J. (2000). "What," "where" and "how" in auditory cortex. Nature Neuroscience, 3, 965-966.

Bosch, V., Mecklinger, A., \& Friederici, A. D. (2001). Slow cortical potentials during retention of object, spatial, and verbal information. Cognitive Brain Research, 10, 219-237.

Bushara, K. O., Weeks, R. A., Ishit, K., Catalan, M.-J., Tian, B., Rauschecker, J. P., \& Hallett, M. (1999). Modality-specific frontal and parietal areas for auditory and visual spatial localization in humans. Nature Neuroscience, 2, 759-766.

CowAn, N. (1999). An embedded-process model of working memory. In A. Miyake \& P. Shah (Eds.), Models of working memory (pp. 62-101). Cambridge: Cambridge University Press.

Cowan, N. (2001). The magical number 4 in short-term memory: A reconsideration of mental storage capacity. Behavioral \& Brain Sciences, 24, 87-114.

Driver, J., \& Spence, C. (1998). Cross-modal attention. Current Opinion in Neurobiology, 8, 245-253.

Goodale, M. A., \& Humphrey, G. K. (1998). The objects of action and perception. Cognition, 67, 181-207.

Goodale, M. A., \& Milner, A. D. (1992). Separate visual pathways for perception and action. Trends in Neurosciences, 15, 20-25.

Greenhouse, S. W., \& Geisser, S. (1959). On methods in the analysis of profile data. Psychometrika, 24, 95-112.

Herrmann, C. S., Senkowski, D., Maess, B., \& Friederici, A. D. (2002). Spatial versus object feature processing in human auditory cortex: A magnetoencephalographic study. Neuroscience Letters, 334, 37-40.

Hommel, B. (1998). Event files: Evidence for automatic integration of stimulus-response episodes. Visual Cognition, 5, 183-216.

Kennett, S., Eimer, M., Spence, C., \& Driver, J. (2001). Tactilevisual links in exogenous spatial attention under different postures: Convergent evidence from psychophysics and ERPs. Journal of Cognitive Neuroscience, 13, 462-478.

Kessels, R. P. C., de Haan, E. H. F., Kappelle, L. J., \& Postma, A. (2001). Varieties of human spatial memory: A meta-analysis on the effects of hippocampal lesions. Brain Research Reviews, 35, 295-303.

Kessels, R. P. C., Kappelle, L. J., de HaAn, E. H. F., \& Postma, A. (2002). Lateralization of spatial-memory processes: Evidence on spatial span, maze learning and memory for object locations. Neuropsychologia, 40, 1465-1473.
Klauer, K. C., \& Stegmaier, R. (1997). Interference in immediate spatial memory: Shifts of spatial attention or central-executive involvement? Quarterly Journal of Experimental Psychology, 50A, 79-99.

LogIE, R. H. (1986). Visuo-spatial processing in working memory. Quarterly Journal of Experimental Psychology, 38A, 229-247.

LogIE, R. H. (1995). Visuo-spatial working memory. Hove, U.K.: Erlbaum.

LogIE, R. H. (2003). Spatial and visual working memory: A mental workspace. In D. E. Irwin \& B. H. Ross (Eds.), The psychology of learning and motivation (Vol. 42, pp. 37-78). Amsterdam: Academic Press.

Logie, R. H., Engelkamp, J., Dehn, D., \& RudKin, S. (2001). Actions, mental actions, and working memory. In M. Denis, R. H. Logie, C. Cornoldi, M. de Vega, \& J. Engelkamp (Eds.), Imagery, language and visuo-spatial thinking (pp. 161-183). Hove, U.K.: Psychology Press.

Logie, R. H., \& Marchetti, C. (1991). Visuo-spatial working memory: Visual, spatial or central executive? In R. H. Logie \& M. Denis (Eds.), Mental images in human cognition (pp. 105-115). Amsterdam: NorthHolland.

Logie, R. H., \& Pearson, D. G. (1997). The inner eye and the inner scribe of visuo-spatial working memory: Evidence from developmental fractionation. European Journal of Cognitive Psychology, 9, 241-257.

Luck, S. J., \& VogeL, E. K. (1997). The capacity of visual working memory for features and conjunctions. Nature, 390, 279-281.

Mazard, A., Tzourio-Mazoyer, N., Crivello, F., Mazoyer, B., \& Mellet, E. (2004). A PET meta-analysis of object and spatial mental imagery. European Journal of Cognitive Psychology, 16, 673-695.

Mecklinger, A., \& Pfeifer, E. (1996). Event-related potentials reveal topographical and temporal distinct neuronal activation patterns for spatial and object working memory. Cognitive Brain Research, 4, 211-224.

Merat, N., \& Groeger, J. A. (2003). Working-memory and auditory localization: Demand for central resources impairs performance. Quarterly Journal of Experimental Psychology, 56A, 531-549.

Merat, N., Groeger, J. A., \& Withington, D. J. (1999). Localizing localization: The role of working memory in auditory localization. International Journal of Psychology, 34, 317-321.

MiddlebrooKs, J. C. (2002). Auditory space processing: Here, there or everywhere? Nature Neuroscience, 5, 824-826.

Paivio, A. (1986). Mental representations: A dual coding approach. New York: Oxford University Press.

Parker, G. J. M., Luzzi, S., Alexander, D. C., Wheeler-Kingshott, C. A. M., Ciccarelli, O., \& Lambon Ralph, M. A. (2005). Lateralization of ventral and dorsal auditory-language pathways in the human brain. NeuroImage, 24, 656-666.

Parmentier, F. B. R., \& Jones, D. M. (2000). Functional characteristics of auditory temporal-spatial short-term memory: Evidence from serial order errors. Journal of Experimental Psychology: Learning, Memory, \& Cognition, 26, 222-238.

Penney, C. G. (1989). Modality effects and the structure of short-term verbal memory. Memory \& Cognition, 17, 398-422.

Postma, A., \& DE HaAn, E. H. F. (1996). What was where? Memory for object locations. Quarterly Journal of Experimental Psychology, 49A, 178-199.

QuinN, J. G., \& McConNell, J. (1999). Manipulation of interference in the passive visual store. European Journal of Cognitive Psychology, 11, 373-389.

Ruchin, D. S., Grafman, J., Cameron, K., \& Berndt, R. S. (2003). Working memory retention systems: A state of activated long-term memory. Behavioral \& Brain Sciences, 26, 709-728.

Salamé, P., \& Baddeley, A. D. (1982). Disruption of short-term memory by unattended speech: Implications for the structure of working memory. Journal of Verbal Learning \& Verbal Behavior, 21, 150-164.

SCHNEIDER, W. X. (1999). Visual-spatial working memory, attention, and scene representation: A neuro-cognitive theory. Psychological Research, 62, 220-236.

Smith, E. E., Jonides, J., Koeppe, R. A., Awh, E., Schuhmacher, E. H., \& Minoshima, S. (1995). Spatial versus object working memory: PET investigations. Journal of Cognitive Neuroscience, 7, 337-356. 
Smith, Z. M., Delgutte, B., \& Oxenham, A. J. (2002). Chimaeric sounds reveal dichotomies in auditory perception. Nature, 416, 87-90.

Smyth, M. M., \& Scholey, K. A. (1994). Interference in immediate spatial memory. Memory \& Cognition, 22, 1-13.

Treisman, A. (1998). The perception of features and objects. In R. Wright (Ed.), Visual attention (Vancouver Studies in Cognitive Science, Vol. 8, pp. 26-54). New York: Oxford University Press.

Treisman, A. (2006). Object tokens, binding and visual memory. In H. D. Zimmer, A. Mecklinger, \& U. Lindenberger (Eds.), Handbook of binding and memory: Perspectives from cognitive neuroscience (pp. 315-338). Oxford: Oxford University Press.

Ungerleider, L. G., Courtney, S. M., \& Haxby, J. V. (1998). A neural system for human visual working memory. Proceedings of the $\mathrm{Na}$ tional Academy of Sciences, 95, 883-890.

Ungerleider, L. G., \& MishKin, M. (1982). Two cortical visual systems. In D. J. Ingle, M. A. Goodale, \& J. W. Mansfield (Eds.), Analysis of visual behavior (pp. 549-586). Cambridge, MA: MIT Press.

Wheeler, M. E., \& Treisman, A. M. (2002). Binding in short-term visual memory. Journal of Experimental Psychology: General, 131, 48-64.

ZIMmer, H. D. (2006). Visuo-spatial working memory: A device also for auditory-spatial memory? Manuscript submitted for publication.

Zimmer, H. D., \& DE VEGA, M. (1996). The medium and the message in spatial communication. In T. Ensik \& C. Sauer (Eds.), Researching technical documents (pp. 153-180). Groningen: University of Groningen.

Zimmer, H. D., \& Speiser, H. R. (2002). The irrelevant picture effect in visuo-spatial working memory: Fact or fiction? Psychologische Beiträge, 44, 223-247.

Zimmer, H. D., Speiser, H. R., \& Seidler, B. (2003). Spatio-temporal working-memory and short-term object-location tasks use different memory mechanisms. Acta Psychologica, 114, 41-65.

\section{NOTES}

1. The 40 objects were an accordion, airplane, alarm clock, bagpipe, banjo, bee, bell, bicycle bell, blackbird, cat, chicken, clock, cow, dog, duck, elephant, frog, goose, guitar, harp, helicopter, horse, lion, mixer, motor saw, motorcycle, owl, phone, piano, pig, police siren, radio, rooster, seagull, sheep, transverse flute, trombone, vacuum cleaner, viola, and wolf.

2. The data of a simple episodic recognition experiment with the same auditory material support this interpretation. The familiar sounds (hits .88) were recognized much better than were the meaningless sounds (.54) $\left[F(1,7)=44.50, M S_{\mathrm{e}}=0.0099, p<.0005\right]$. Despite the differing experimental paradigms, this result indicates that performance in the spatial task, too, suffered mainly from worse memory for nonsense stimuli (the objects), and not from worse object-to-position assignment.

3. Of course, mixed-list auditory items also benefited from the fact that this condition reduced spatial uncertainty even further.

(Manuscript received February 27, 2004; revision accepted for publication June 25,2005 .) 